\title{
Changes in Quasi-periodic Variations of Solar Photospheric Fields: Precursor to the Deep Solar Minimum in Cycle 23?
}

\section{Susanta Kumar Bisoi, P. Janardhan, D. Chakrabarty, S. Ananthakrishnan \& Ankur Divekar}

\section{Solar Physics}

A Journal for Solar and Solar-Stellar Research and the Study of Solar Terrestrial Physics

ISSN 0038-0938

Volume 289

Number 1

Sol Phys (2014) 289:41-61

DOI 10.1007/s11207-013-0335-3

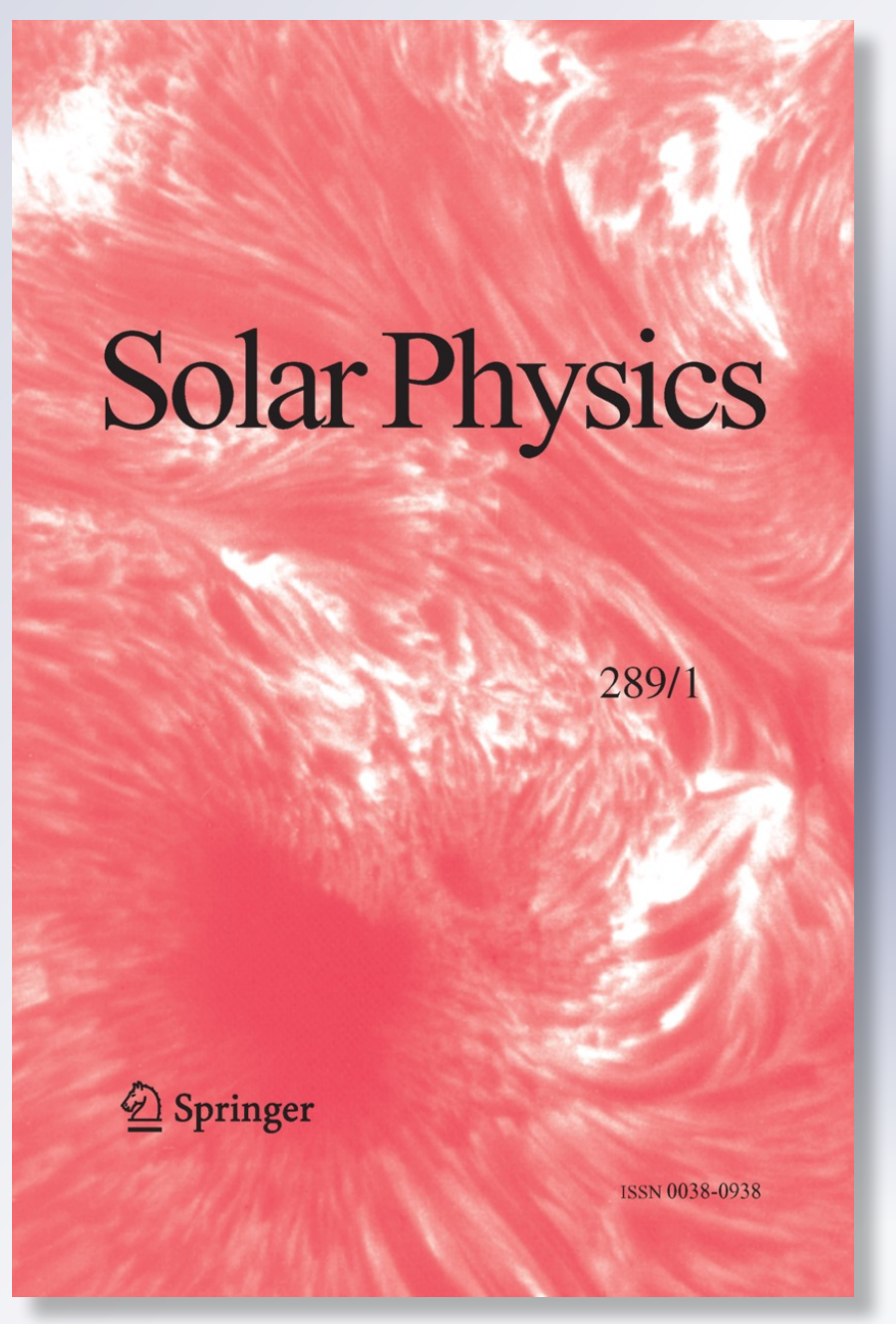

黛 Springer 
Your article is protected by copyright and all rights are held exclusively by Springer Science +Business Media Dordrecht. This e-offprint is for personal use only and shall not be selfarchived in electronic repositories. If you wish to self-archive your article, please use the accepted manuscript version for posting on your own website. You may further deposit the accepted manuscript version in any repository, provided it is only made publicly available 12 months after official publication or later and provided acknowledgement is given to the original source of publication and a link is inserted to the published article on Springer's website. The link must be accompanied by the following text: "The final publication is available at link.springer.com". 


\title{
Changes in Quasi-periodic Variations of Solar Photospheric Fields: Precursor to the Deep Solar Minimum in Cycle 23?
}

\author{
Susanta Kumar Bisoi • P. Janardhan • D. Chakrabarty • \\ S. Ananthakrishnan • Ankur Divekar
}

Received: 15 November 2012 / Accepted: 20 May 2013 / Published online: 13 July 2013

(C) Springer Science+Business Media Dordrecht 2013

\begin{abstract}
Possible precursor signatures in the quasi-periodic variations of solar photospheric fields were investigated in the build-up to one of the deepest solar minima experienced in the past 100 years. This unusual and deep solar minimum occurred between Solar Cycles 23 and 24. We used both wavelet and Fourier analysis to study the changes in the quasi-periodic variations of solar photospheric fields. Photospheric fields were derived using ground-based synoptic magnetograms spanning the period 1975.14 to 2009.86 and covering Solar Cycles 21, 22, and 23. A hemispheric asymmetry in the periodicities of the photospheric fields was seen only at latitudes above $\pm 45^{\circ}$ when the data were divided into two parts based on a wavelet analysis: one prior to 1996 and the other after 1996. Furthermore, the hemispheric asymmetry was observed to be confined to the latitude range of $45^{\circ}$ to $60^{\circ}$. This can be attributed to the variations in polar surges that primarily depend on both the emergence of surface magnetic flux and varying solar-surface flows. The observed asymmetry along with the fact that both solar fields above $\pm 45^{\circ}$ and micro-turbulence levels in the
\end{abstract}

S.K. Bisoi $(\bowtie) \cdot$ P. Janardhan

Astronomy \& Astrophysics Division, Physical Research Laboratory, Navrangpura, Ahmedabad 380009, India

e-mail: susanta@prl.res.in

P. Janardhan

e-mail: jerry@prl.res.in

D. Chakrabarty

Space \& Atmospheric Sciences Division, Physical Research Laboratory, Navrangpura, Ahmedabad 380009, India

e-mail: dipu@prl.res.in

S. Ananthakrishnan · A. Divekar

Department of Electronic Science, University of Pune, Pune, Maharasthra 411007, India

S. Ananthakrishnan

e-mail: subra.anan@gmail.com

A. Divekar

e-mail: wiztronix@gmail.com 
inner-heliosphere have been decreasing since the early- to mid-nineties (Janardhan et al. in Geophys. Res. Lett. 382, 20108, 2011) suggest that around this time active changes occurred in the solar dynamo that governs the underlying basic processes in the Sun. These changes in turn probably initiated the build-up to the very deep solar minimum at the end of Cycle 23 . The decline in fields above $\pm 45^{\circ}$, for well over a solar cycle, would imply that weak polar fields have been generated in the past two successive solar cycles, viz. Cycles 22 and 23. A continuation of this declining trend beyond 22 years, if it occurs, will have serious implications for our current understanding of the solar dynamo.

Keywords Magnetic fields, photosphere - Solar cycle, observations · Solar periodicity · Surges

\section{Introduction}

The delayed onset of Solar Cycle 24, after one of the deepest solar minima experienced in the past 100 years, has had significant solar and heliospheric consequences. Solar Cycle 23 has been characterized by a steady decline in solar activity (McComas et al., 2008; Jian, Russell, and Luhmann, 2011), a continuous weakening of polar fields (Jiang et al., 2011), and a decline in micro-turbulence levels in the inner heliosphere since $\approx 1995$ (Janardhan et al., 2011). Investigations of the boundary of polar coronal holes during the declining phase of Solar Cycle 23 using images from the Extreme Ultraviolet Imaging Telescope (EIT) onboard the Solar and Heliospheric Observatory ( $\mathrm{SoHO}$ ) have found a decrease in coronal hole area by $\approx 15 \%$ in comparison to that observed at the beginning of Solar Cycle 23 (Kirk et al., 2009). Using both ground-based and space-borne observations of photospheric magnetic fields (Janardhan, Bisoi, and Gosain, 2010; Hathaway and Rightmire, 2010) and theoretical modeling (Dikpati et al., 2010; Nandy, Muñoz-Jaramillo, and Martens, 2011), there have been continuous efforts to investigate and understand the behavior of solar photospheric fields and their correlation with meridional flows to try to explain the delayed onset of Cycle 24 and the cause of the deep minimum at the end of Cycle 23.

Magnetic-field measurements using data from the National Solar Observatory, Kitt Peak (NSO/KP) synoptic magnetogram database, have shown that a decline in solar photospheric fields in the latitude range of $45^{\circ}$ to $78^{\circ}$ began around the minimum of Solar Cycle 22, in $\approx 1995-1996$ (Janardhan, Bisoi, and Gosain, 2010). However, the dipole field of the Sun behaved differently. It was at its strongest in 1995, weakened at solar maximum around 2000, and then increased between $\approx 2000$ and 2004 (Wang, Robbrecht, and Sheeley, 2009; Janardhan, Bisoi, and Gosain, 2010). Signatures of this decline in solar fields above latitude $\pm 45^{\circ}$ have also been observed in the inner heliosphere as a corresponding decline in micro-turbulence levels, which in turn correspond to small-scale interplanetary magnetic fields (Ananthakrishnan, Coles, and Kaufman, 1980; Ananthakrishnan, Balasubramanian, and Janardhan, 1995). The decline in micro-turbulence levels was inferred from extensive interplanetary scintillation (IPS) observations at $327 \mathrm{MHz}$ in the period 1983-2009 (Janardhan et al., 2011). Because both solar magnetic fields and micro-turbulence levels in the inner-heliosphere have been declining since mid-1990, Janardhan et al. (2011) have argued that the build-up to the deep and extended solar minimum at the end of Cycle 23 was initiated as early as the mid-1990.

One method of gaining insights into underlying basic processes in the solar interior that govern the nature and evolution of solar photospheric magnetic fields is studying the periodicities produced by various surface-activity features at different times in the solar cycle. For 
example, the 158-day Rieger periodicity reported in the solar-flare occurrence rates (Oliver, Ballester, and Baudin, 1998) has been linked to the periodic emergence of magnetic flux through the photosphere, which in turn gives rise to a periodic variation of the total sunspot area on the solar surface. Similarly, a strong 1.3-year periodicity detected at the base of the solar convection zone in a helioseismic study (Howe et al., 2000) has also been detected in sunspot areas and sunspot number time series studied using wavelet transforms (Krivova and Solanki, 2002). Krivova and Solanki have proposed that the 154-158-day Rieger period is a harmonic of the 1.3 -year periodicity $(3 \times 156$ days $=1.28$ years $)$ and that variations in the rotation rate have a strong influence on the workings of the solar dynamo (Krivova and Solanki, 2002).

To understand the role of periodic changes, if any, in the solar photospheric fields that led to the build-up of the recent deep minimum at the end of Cycle 23, data from the NSO/KP synoptic magnetic database during the period 1975.14-2009.86 were subjected to detailed spectral analyses using both wavelet and Fourier techniques. It has been shown that a significant North - South asymmetry in the quasi-periodic variations of the photospheric magnetic fields exist prior to and after 1996 (with 1996 being a clear transition period) almost 12 years before the deep minimum period in 2008-2009.

\subsection{Solar Periodicities}

Prominent periodicities related to the solar cycle, in addition to the well-known synodic rotation period of $\approx 27$ days and the sunspot activity cycle of $\approx 11$ years, have been a topic of interest for over two decades now. Using data from the Gamma Ray Spectrometer (Forrest et al., 1980) onboard the Solar Maximum Mission, a clear 154-day periodicity in the occurrence rate of high-energy $(0.3-100 \mathrm{MeV})$ flares has been reported (Rieger et al., 1984). This was also confirmed at other wavelengths (Verma and Joshi, 1987; Droege et al., 1990; Kile and Cliver, 1991; Chowdhury and Ray, 2006), and for solar energetic-particle events (Lean, 1990; Krivova and Solanki, 2002; Kiliç, 2008; Chowdhury, Khan, and Ray, 2010; Chowdhury and Dwivedi, 2011).

In addition, other intermediate periodicities with periods ( $<$ one year) have also been reported, viz. a 51-day periodicity in the comprehensive flare index (Bai, 1987), a 127day periodicity in the 10-cm radio flux (Kile and Cliver, 1991), periodicities of 33.5 days, 51 days, 84 days, 129 days, and 153 days in the solar-flare occurrence during different phases of Solar Cycles 19-23 (Bai, 2003), periodicities of 100-103 days, 124-129 days, $151-158$ days, $177 \pm 2$ days, 209-222 days, $232-249$ days, $282 \pm 4$ days, $307-349$ days in the unsigned photospheric fluxes (Knaack, Stenflo, and Berdyugina, 2004, 2005) and periodicities of 87 - 106 days, 159- 175 days, 194-219 days, 292-318 days for Cycle 22, and 69 - 95 days, 113 - 133 days, 160 - 187 days, 245 - 321 days, 348 - 406 days in sunspot areas at different phases of Cycle 23 (Chowdhury, Khan, and Ray, 2009).

For periodicities, between one and five years, a 1.3-year periodicity in sunspot areas and numbers (Krivova and Solanki, 2002), a 1-year and 1.7-year periodicity in solar total and open fluxes (Mendoza, Velasco, and Valdés-Galicia, 2006), a 1.3-year, 1.43-year, 1.5-year, 1.8-year, 2.4-year, 2.6-year, 3.5-year, 3.6-year, and 4.1-year periodicity in unsigned photospheric fluxes (Knaack, Stenflo, and Berdyugina, 2004, 2005) have been reported. Studies of all these periodicities have shown an asymmetry in solar-activity phenomena between the northern and southern hemispheres of the Sun. This hemispheric asymmetry, on different time scales and at different phases of different solar cycles, has been observed in various types of solar activity (Howard, 1974; Verma, 1987; Oliver and Ballester, 1994; Knaack, Stenflo, and Berdyugina, 2005) and has been providing invaluable information 
about basic underlying physical processes on the Sun. Since processes such as differential rotation of the Sun and emergence of magnetic flux determine the strength and distribution of solar magnetic activity, investigating solar-cycle-related periodic or quasi-periodic variations is crucial for understanding the behavior and nature of solar magnetic fields.

\section{Decline in Mid- to High-Latitude Solar Fields}

Figure 1 shows temporal variation in the unsigned, or absolute, solar photospheric magnetic fields in the latitude range of $45^{\circ}-78^{\circ}$ in both solar hemispheres for the period from February 1975 to November 2009 (panel (a)) covering Solar Cycles 21, 22, and 23. The vertically oriented dotted lines demarcate the period of solar minimum of these three solar cycles. The small, black filled dots and the open circles are the actual measurements for the northern and southern hemispheres, while the solid red (northern hemisphere) and blue (southern hemisphere) lines are smoothed curves derived using a robust Savitzky-Golay (SG) algorithm (Savitzky and Golay, 1964) that can preserve features of the input distribution such as maxima and minima while effectively suppressing noise. These features are generally flattened by other smoothing techniques. The decline in photospheric magnetic fields in both hemispheres, starting around the early- to mid-1990s, can be clearly seen in Figure 1. While the decline in photospheric fields had started in $\approx 1991$ for the northern hemisphere (solid red curve), for the southern hemisphere the decline started in $\approx 1995$ (solid blue curve). Beyond $\approx 1996$, a steady decline in the photospheric fields for both hemispheres can be clearly seen. A similar decline in the sunspot umbral magnetic field strength since $\approx 1998$ has been reported as well (Livingston, 2002; Penn and Livingston, 2006). Also shown in the bottom two panels of Figure 1 are the magnetic residuals, obtained by subtracting the SG fit from the actual measurements for the two hemispheres in the latitude range of $45^{\circ}-78^{\circ}$ (panel (b)) and $0^{\circ}-45^{\circ}$ (panel (c)) respectively. See the upper two panels of Figure 1 in Janardhan, Bisoi, and Gosain (2010) for measurements of the magnetic field in the range of $\pm 45^{\circ}$, which show a strong solar-cycle modulation in the data, as expected.

The main details of the method for deriving the magnetic fields are given below. We used measurements of magnetic-field strengths obtained from 466 individual Carrington rotations (CR) of NSO/KP synoptic magnetograms from CR 1625 through CR 2090 in the period from 19 February 1975 to 09 November 2009 (1975.14-2009.86). Each magnetogram, generated from daily ground-based full-disk magnetograms spanning a Carrington rotation period (27.27 days), was longitudinally averaged to a $1^{\circ}$-wide longitudinal strip covering the latitude range from $-90^{\circ}$ to $+90^{\circ}$. Surface magnetic fields in both equatorial $\left(-45^{\circ}\right.$ to $+45^{\circ}$ ) and high-latitude zones ( $>45^{\circ}$ in both hemispheres) of the Sun were then derived by averaging over appropriate latitude regions. Full details of the method are described by Janardhan, Bisoi, and Gosain (2010).

The SG smoothing was performed so that the resulting residual time-series is stationary: i.e. the mean of time series is or approaches zero. This detrending of the data removes strong periodic variations $(\approx 11$ years). The residuals were then subjected to a wavelet and a Fourier time-series analysis to study temporal periodic variations in the photospheric magnetic fields. In the remainder of the text the fields obtained in the equatorial belt of $-45^{\circ}$ to $+45^{\circ}$ are referred to as low-latitude fields, while the fields obtained at latitudes $>45^{\circ}$ in the two hemispheres are referred to as high-latitude fields. 
Figure 1 (a) Measurements of solar photospheric magnetic fields in the latitude range of $45^{\circ}$ to $78^{\circ}$. The filled dots and the open circles represent the measurements for the northern and southern hemispheres while the fit obtained using the SG algorithm is shown by the solid red (North) and blue (South) lines. The vertically oriented dotted lines demarcate the periods of Solar Cycles 21, 22, and 23. (b) The residuals obtained after subtracting the SG-smoothed values from the magnetic fields in the latitude range $45^{\circ}$ to $78^{\circ}$ are shown for the North and South. (c) The residual fields for the northern and southern hemispheres obtained from the magnetic fields at latitudes below $45^{\circ}$.
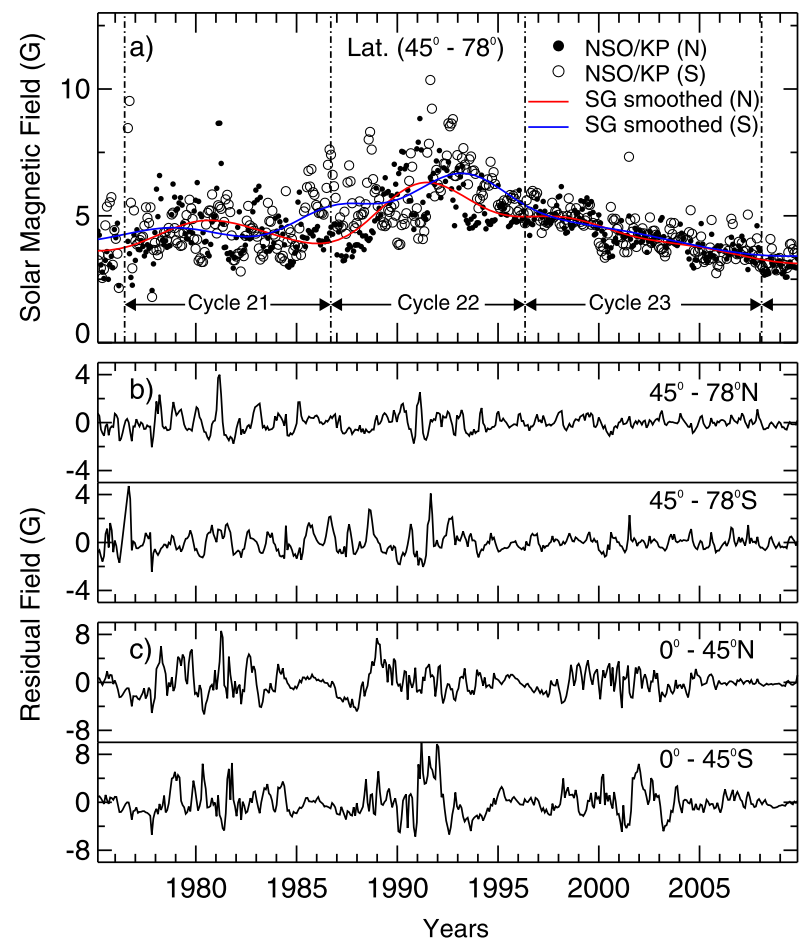

\subsection{Transition in Wavelet Periodicities}

A wavelet transform is basically the convolution of a time series with the scaled and translated version of a chosen mother wavelet function. Wavelet analysis now is frequent used in the analysis of time-series data since it yields information in both time and frequency domains (Torrence and Compo, 1998). Using a Morlet wavelet as the mother wavelet and based on the algorithm by Torrence and Compo (1998), the magnetic residuals obtained for the high- and low-latitude fields in the period from 19 February 1975 to 09 November 2009 (1975.14-2009.86) were subjected to a wavelet analysis. Scattered data gaps amounting to $\approx 2.5 \%$ of the data were replaced with values obtained using a cubic-spline interpolation. The Morlet wavelet function $\psi_{0}(\eta)$, represented in Equation (1) below, is a plane wave modulated by a Gaussian,

$$
\psi_{0}(\eta)=\pi^{-1 / 4} \mathrm{e}^{\mathrm{i} \omega_{0} \eta} \mathrm{e}^{-\eta^{2} / 2}
$$

Here, $\omega_{0}$ is a non-dimensional frequency that determines the number of oscillations in the wavelet, and $\eta$ is a non-dimensional time parameter. The choice of different nondimensional frequencies defines the frequency resolution. However, by varying the frequency resolution, one has to compromise with temporal resolution. In this case, we chose $\omega_{0}$ to be six to obtain a better frequency resolution. This value was left unchanged and was not tuned to different values because our interest is to look for changes in the frequency components rather than to find precisely the low- and high-frequency components. However, in the following sections, we employed a Fourier-analysis technique to find the various frequency components. 


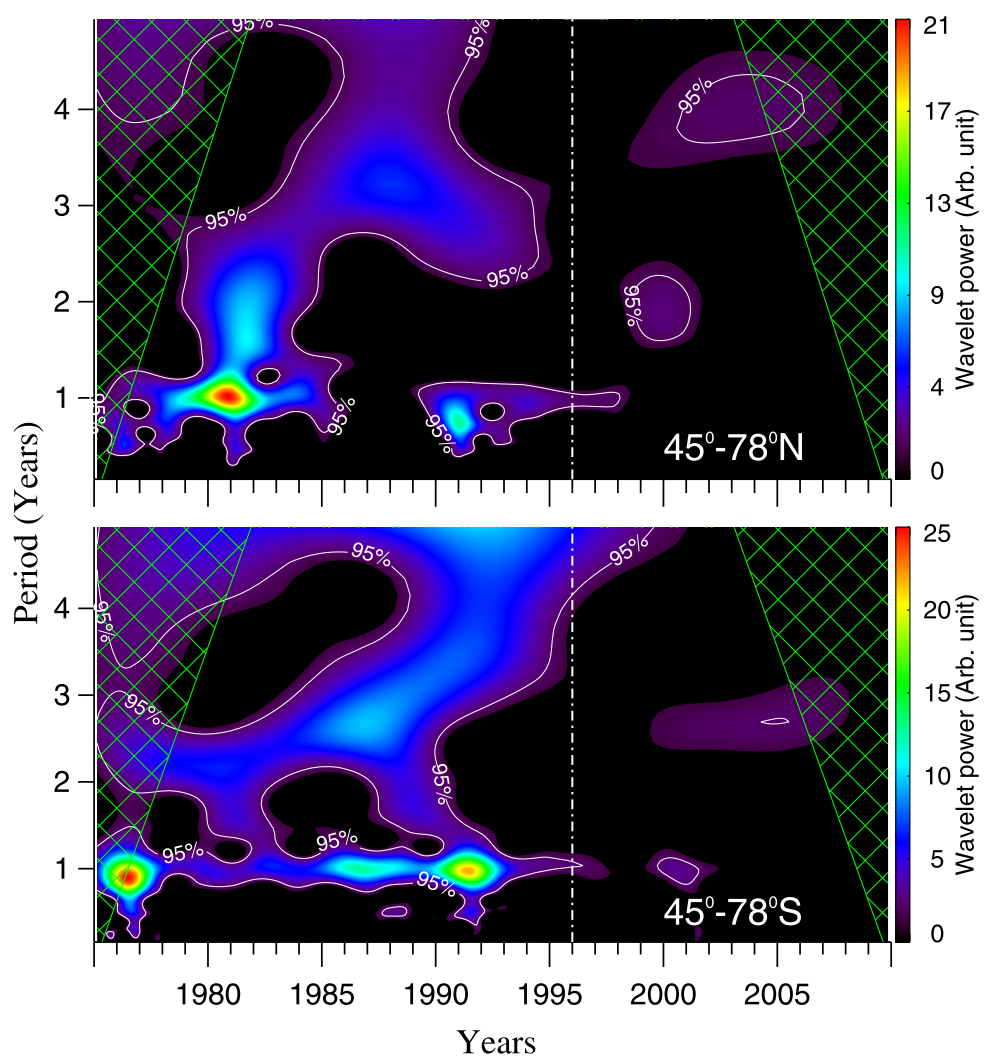

Figure 2 The wavelet-power distribution of the solar periodicity over years for high-latitude fields for the northern (upper panel) and southern hemisphere (lower panel) in the latitude range of $45^{\circ}$ to $78^{\circ}$. The green cross-hatched regions are the cone of influence (COI), the white contours indicate a significance level at $95 \%$. The dashed vertical lines are marked at 1996 when a clear transition has been observed in the wavelet power of the periodicities for the North and South high-latitude fields.

Figure 2 shows the wavelet spectrum for the high-latitude fields in the northern (upper panel) and southern hemisphere (lower panel), while Figure 3 shows the wavelet spectrum for the low-latitude fields in the northern (upper panel) and southern hemispheres (lower panel). For the high-latitude fields (Figure 2) in the North and South, significant wavelet power is only seen at lower periods $(\approx$ one to three years) prior to 1985 , while significant wavelet power is seen at lower and higher periods $(\approx$ three to five years) for the interval between 1985 and 1996. For the low-latitude fields (Figure 3), we see no significant changes in the power levels between 1975 and 1996.

It is important to note here that there is a clear transition in periodicities and power levels in the two hemispheres around 1996 for high- and low-latitude fields. We stress here that we carefully checked that selecting different colors or changes in the color intensity in the wavelet plots does not change the transition noticed in the wavelet-power spectra. Although the decline in the fields has started in the early- to mid-1990, as seen from Figure 1, the wavelet spectra show that there is an unambiguous transition in the quasi-periodic variations of fields in the two hemispheres at $\approx 1996$. We therefore divided in the remaining analysis the residuals into two sets based on this transition in the wavelet spectra around 1996 to 


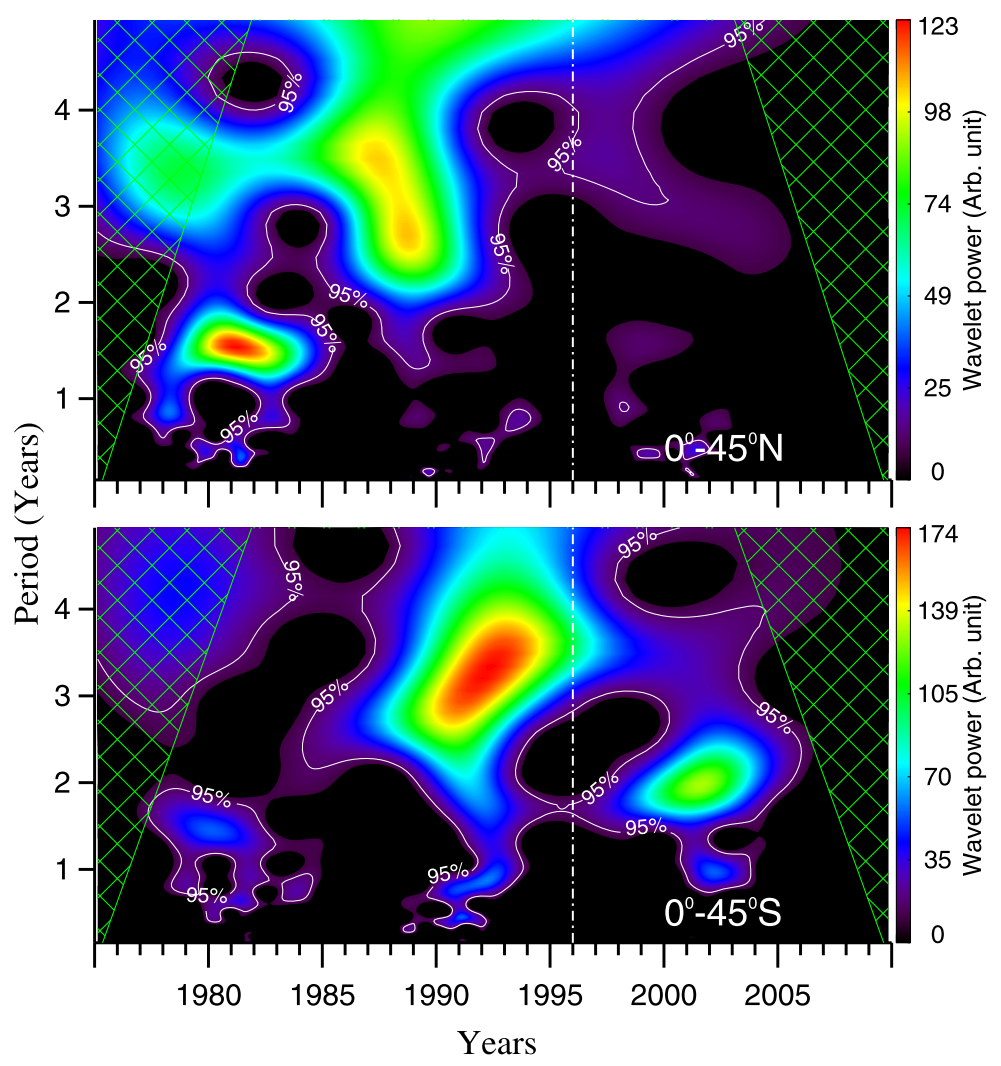

Figure 3 The wavelet-power distribution of the solar periodicity over years for low-latitude fields for the northern (upper panel) and southern hemisphere (lower panel) in the latitude range of $0^{\circ}$ to $45^{\circ}$. The green cross-hatched regions are the cone of influence (COI), the white contours indicate a significance level at $95 \%$. The dashed vertical line demarcates the period of transition in wavelet power of the periodicities for the two hemispheres around 1996.

study the changes, if any, in the periodicities before and after this transition. A Fourier timeseries analysis was then carried out separately on the residuals used in the wavelet analysis for the period prior to 1996 and the period after 1996 for the high- and low-latitude fields.

It is important to bear in mind here that the transition in the wavelet spectra around 1995 1996 corresponds to the time when the solar high-latitude fields above $\pm 45^{\circ}$ and solar wind turbulence levels in the entire inner heliosphere began to decline (Janardhan, Bisoi, and Gosain, 2010; Janardhan et al., 2011).

\section{Fourier Periodicities - Asymmetries and Symmetries}

Based on the transition seen in the wavelet spectra at $\approx 1996$, we subjected the residuals obtained from the SG fits to a Fourier analysis after dividing the time series of the residuals, spanning years 1975.14 to 2009.86, into two parts, one prior to 1996 and the other after 1996. Since the time series of the residual field has some data gaps amounting to $\approx 2.5 \%$ of the data, the algorithm used for this purpose was based on the Lomb-Scargle Fourier transform for unevenly spaced data (Lomb, 1976; Scargle, 1982, 1989; Schultz and Stattegger, 1997) in 


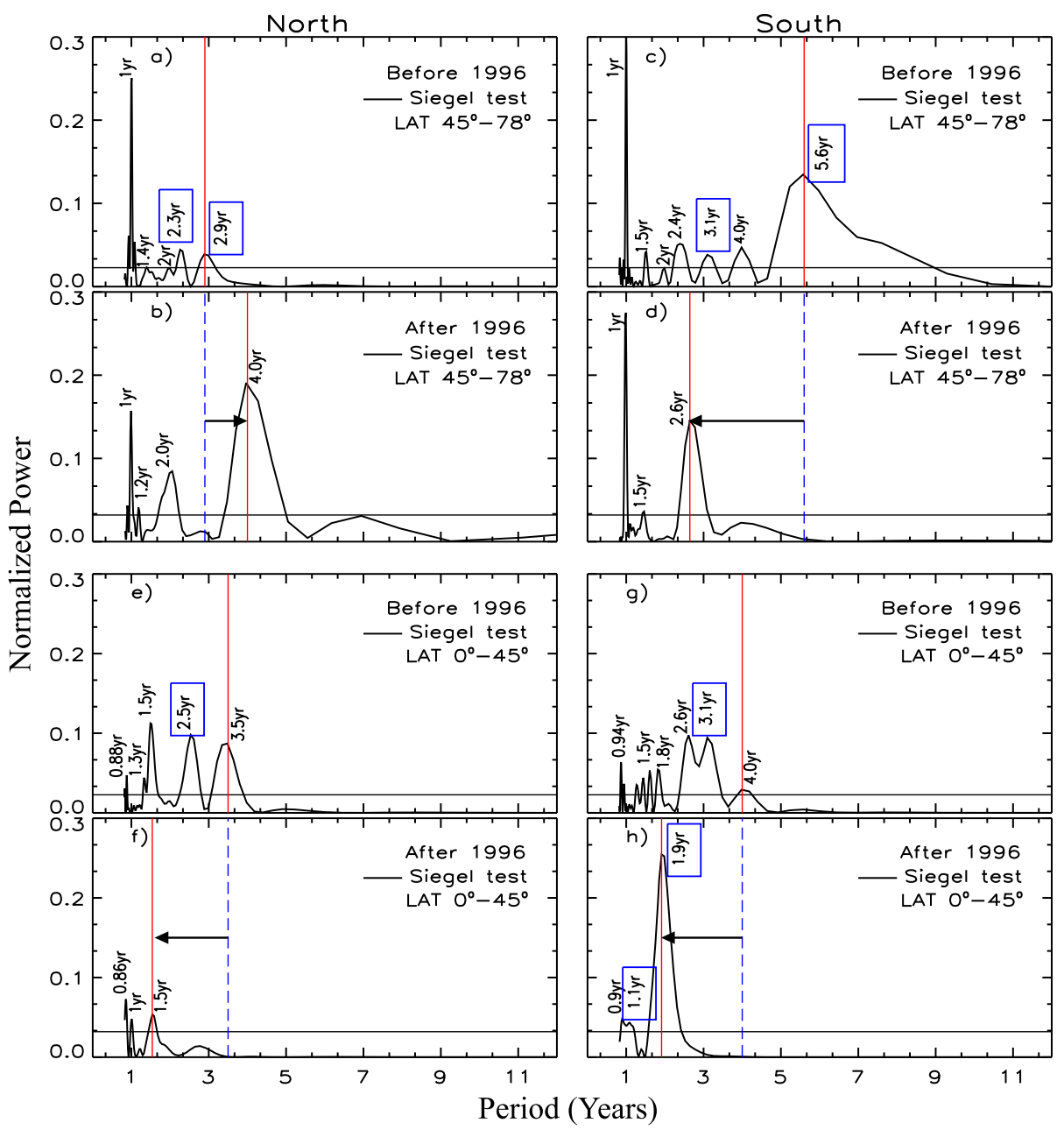

Figure 4 Normalized Fourier power distribution with the periodicity for photospheric fields in the North (left column) and South (right column) before [(a), (c), (e), (g)] and after [(b), (d), (f), (h)] 1996. The solid-red vertical lines demarcate the highest period in each of the four panels, the dotted-blue vertical line and direction of the black arrow in each panel are used to show the shift in the periodicity of the longest periods. The solid-horizontal lines depict significance levels as determined by the Siegel test. The new periodicities from our analysis are boxed in blue.

combination with the Welch-Overlapped-Segment-Averaging (WOSA) procedure (Welch, 1967). The spectral power derived from this analysis is generally normalized with respect to the total power contained in all the Fourier components taken together. This procedure makes the relative distribution of the spectral power independent of the spectral windowing used in the algorithm. Such normalized power spectra are referred to as normalized Fourier periodograms, or simply normalized periodograms.

\subsection{High-Latitude Fields}

Normalized periodograms obtained from the Fourier analysis are shown in Figure 4. Figures 4(a)-(d) represent the high-latitude fields, Figures 4(e)-(h) represent the low- 
latitude fields. The upper four panels (high-latitude fields) show the normalized periodograms before and after 1996 in the North (panels (a) and (b)), and before and after 1996 in the South (panels (c) and (d)). In a similar fashion, the lower four panels (low-latitude fields) show the normalized periodograms in the North before and after 1996 (panels (e) and (f)) and in the South before and after 1996 (panels (g) and (h)). The significant periodic components were determined using the Siegel test statistics (Siegel, 1980); these are the components with power levels above the black horizontal line drawn in each panel of Figure 4. The Siegel test is an extension of the Fisher test (Fisher, 1929). While the Fisher test attempts to spot the single dominant periodicity in a time series that has maximum power in the periodogram and is above the critical level defined by the Fisher-test statistics, the Siegel test relaxes this stringent condition a little and considers two to three dominant periodic components above the critical level. These test statistics and the process of determining the confidence/significance level are discussed by Percival and Walden (1993) and also briefly by Schultz and Stattegger (1997). We chose a $95 \%$ confidence level (or $5 \%$ significance level). This is equivalent to the $\pm 2 \sigma$ level in the FFT method. The choice of this confidence level decides the critical level of the Siegel-test statistics (Schultz and Stattegger, 1997).

Although the Fourier components obtained from the analysis range in periods from 54 days to 12 years corresponding to $214 \mathrm{nHz}$ to $2.6 \mathrm{nHz}$ in frequency, Figure 4 only shows the components with periods in the range 300 days to 12 years, i.e. the low-frequency range. Normalized periodograms containing Fourier components with periods shorter than 300 days are discussed later.

The red vertical lines in each panel of Figure 4 are drawn through the peak of the component with the longest period. A shift in the periodicities can be seen after 1996 when compared to those before 1996. It must be emphasized that we are not interested in the actual periodicities themselves, but rather in the manner in which the longest periodicities shift between the time interval prior to and after 1996. It can be seen from Figure 4 that, in addition to the shift in periodicity of the high-latitude fields in the two hemispheres before and after 1996, the spectral power also differs significantly before and after 1996. The longer periods after 1996 have more spectral power than the longer periods prior to 1996 . If we denote the longest periods for the high-latitude fields in the North before 1996 as $T_{1}$ and that after 1996 as $T_{2}$, we find that $T_{2}>T_{1}$ for high-latitude fields in the North.

For the southern hemisphere, the shift in periodicities, as indicated by the direction of the black arrow in Figure 4, is in the opposite direction from the northern hemisphere. If we denote the longest periods for the high-latitude fields in the South before 1996 and after 1996 as $T_{3}$ and $T_{4}$ respectively, we find that $T_{4}<T_{3}$ for the high-latitude fields in the South. As for the northern hemisphere, the distribution of spectral power in various periodicities is also significantly different in the periods before and after 1996 .

Thus, a North-South asymmetry is seen in the distribution of periodicities for the highlatitude fields along with significant changes in the power of various periodic components. Since the normalized periodograms are generated from measurements of magnetic fields, it is a proxy for the behavior of the photospheric magnetic fields and a North-South asymmetry would imply a similar asymmetry in photospheric magnetic fields toward higher solar latitudes before and after 1996.

\subsection{Low-Latitude Fields}

In contrast to the behavior of the high-latitude fields, the low-latitude fields shown in Figure $4(\mathrm{e})-(\mathrm{h})$ show no asymmetry in the manner in which the longest periods shift in 
the periodograms prior to and after 1996. The shift in the longest periods in the North and South low-latitude fields before and after 1996 is depicted in the lower four panels of Figure 4 in a similar fashion to that of the upper four panels. The vertical solid-red line is drawn through the peak of the component with the longest period, and the dotted-blue line and a black arrow in each panel indicate the direction of the shift.

Denoting the longest period for the low-latitude fields in the North before and after 1996 as $T_{5}$ and $T_{6}$, respectively, we find that $T_{5}>T_{6}$. In the southern hemisphere, denoting the longest period for the low-latitude fields prior to 1996 and after 1996 as $T_{7}$ and $T_{8}$, respectively, it is clear that $T_{7}>T_{8}$. Hence, the longest periods show a shift in the same direction, indicating that there is no asymmetry in the distribution of the low-latitude fields before and after 1996.

\section{Latitudinal Profile for the Fourier Power Spectrum}

To examine how the Fourier spectrum for the high-latitude fields varies as a function of latitude, the residuals for the high-latitude fields were subdivided into $15^{\circ}$-wide latitude bins and a Fourier analysis was carried out for the data in each latitude bin. This was done to examine the changes, if any, in the spectral distribution with latitude and because the strength and distribution of photospheric magnetic fields changes with solar latitudes. It would therefore be interesting to see if the North-South asymmetry in the high-latitude fields persists across all latitude bins. Figure 5 shows normalized periodograms in the latitude range $30^{\circ}$ to $75^{\circ}$ in latitude steps of $15^{\circ}$. Figure $5(\mathrm{a})-(\mathrm{d}),(\mathrm{e})-(\mathrm{h})$, and (i) $-(\mathrm{k})$ represent normalized periodograms showing the distribution of Fourier periodicities in latitude ranges of $30^{\circ}-$ $45^{\circ}, 45^{\circ}-60^{\circ}$, and $60^{\circ}-75^{\circ}$. The latitude ranges are indicated at the top right-hand corner of each panel. Starting from the top, each of the left-column panels of Figure 5 represents the power spectra for the northern hemisphere in each latitude bin in pairs before and after 1996. Similarly, the right-column panels of Figure 5 represent power spectra obtained for each latitude bin in the southern hemisphere in pairs before and after 1996. It can be seen from Figure 5 that the North-South asymmetry is present only in the $45^{\circ}-60^{\circ}$ bin. Although this is not shown in Figure 5, we verified that the asymmetry is also absent from the latitude bins of $0^{\circ}$ to $15^{\circ}$ and $15^{\circ}$ to $30^{\circ}$.

In the $60^{\circ}-75^{\circ}$ bin (bottom four panels of the Figure 5), the shift of the longest periods before and after 1996 shows no North-South asymmetry. However, the spectral powers in the longest periods are significantly different before and after 1996. The spectral power in the longest-period component increases significantly after 1996 for the northern hemisphere, but decreases for the southern hemisphere.

It is important to note here that the latitude band $45^{\circ}-60^{\circ}$ in both hemispheres is dominated by surges or tongues of magnetic flux that is carried poleward by the meridional flow. These surges are a direct surface manifestation of the meridional flow, which is in turn governed by an internal solar dynamo. Beyond $60^{\circ}$ in latitude, the high-latitude flux in both hemispheres saturates and can be best seen along with the magnetic surges in the $45^{\circ}-60^{\circ}$ latitude band in a magnetic butterfly-diagram, which is described in the following section.

\subsection{The Magnetic Butterfly-Diagram and Polar Surges}

It is generally believed that the emergence and evolution of a magnetic field in the solar photosphere is tied to a solar dynamo in the solar interior. In particular, the toroidal field manifests itself as solar-surface bipolar active regions with sunspots migrating toward the 


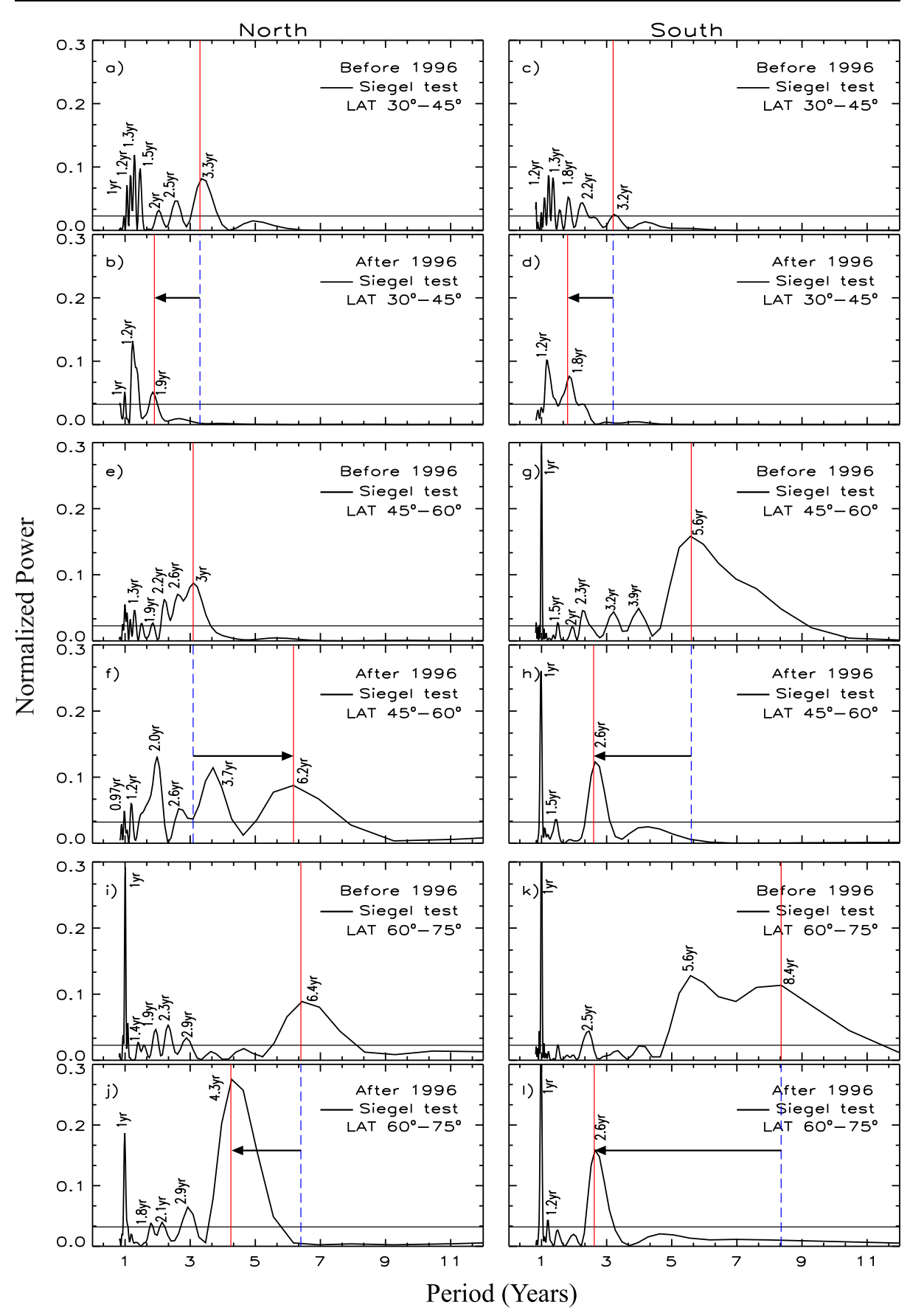

Figure 5 Normalized Fourier periodograms in the North (left column) and South (right column) before [(a), (c), (e), (g), (i), (k)] and after [(b), (d), (f), (h), (j), (l)] 1996 for the photospheric fields in latitude ranges of $30^{\circ}-45^{\circ}, 45^{\circ}-60^{\circ}$, and $60^{\circ}-75^{\circ}$. The solid-red vertical line, the dotted-blue vertical line, and the black arrow in each panel are used to show the shift in periodicity and the spectral power of the fields before and after 1996 in the northern and southern hemisphere. The solid horizontal lines depict significance levels as determined by the Siegel test. 
Equator as the solar cycle progresses. This migration gives rise to the well-known butterfly diagram, which is basically a map of longitudinally averaged magnetic fields in time. Solardynamo models that explain the main features of solar magnetic activity consider the strong toroidal field as being generated by the shearing of a pre-existing weak poloidal field by solar differential rotation ( $\omega$-effect). Subsequently, the regeneration and inversion of polar fields, at the maximum of each solar cycle, is caused by the cancellation of sunspot fields at the Equator along with a net poleward surface flux that is transported via meridional circulation to reach the poles and reverse the pre-existing polar fields (Babcock-Leighton type- $\alpha$ effect).

We generated a butterfly diagram from the NSO/Kitt Peak synoptic maps by using longitudinally averaged photospheric fields derived from the synoptic maps described in Section 2. Figure 6(a) shows a butterfly diagram for Solar Cycles 21, 22, and 23 covering the period from 19 February 1975 to 09 November 2009 (1975.14-2009.86). The leading and following polarity fluxes in Figure 6(a) are shown in red and green for the northern hemisphere in Cycle 21, with the derived flux values indicated in a color-coded bar at the top of the figure. Leading and following polarity fluxes will be reversed in the southern hemisphere and will again flip from cycle to cycle and hemisphere to hemisphere.

To obtain better contrast, the fluxes in Figure 6(a) were limited to the range $\pm 30 \mathrm{G}$. The poleward motion of the trailing polarity fluxes above the latitudes $\pm 45^{\circ}$, known as polar surges, are episodic in nature (Wang, Nash, and Sheeley, 1989) and can be clearly seen as tongues of red and green bands in Figure 6(a). For a better view of the polar surges, the middle and lower panels of Figure 6 show variations of intensity as a function of time in each $1^{\circ}$ strip of latitude in the latitude range $45^{\circ}-60^{\circ}$ for the northern (Figure 6(b)) and southern hemisphere (Figure 6(c)). The solar field for each degree of latitude is shown after smoothing over three Carrington rotations. An example of a surge in the North and South is highlighted in red.

Knaack, Stenflo, and Berdyugina, 2005 reported a periodicity of 1.3 years in the photospheric fields that is correlated with these polar surge motions. We see an approximate periodicity of one year in the polar surge flows in the lower two panels of Figure 6. This agrees with the strong one-year periodicity seen in Figure 4 and 5. Furthermore, a variation in the strength and occurrence rate of surges prior to and after 1996 can be seen from a careful inspection of Figure 6. In the latitude bin $\pm 45^{\circ}- \pm 60^{\circ}$, we find that the frequency of these surges is greater during the years 1996-2009, while the surges are less frequent during the years $1986-1995$.

\section{Fourier Periodicity - Low- and High-Frequency Periods}

The harmonic analysis yielded Fourier components with periods ranging from 54 days to 12 years, corresponding to a frequency range of $2.6 \mathrm{nHz}$ to $214 \mathrm{nHz}$. The significant periodic components, as determined by the Siegel test, were grouped into low-frequency periods in the frequency range $38.5 \mathrm{nHz}$ ( 300 days) to $2.6 \mathrm{nHz}$ (12 years) and high-frequency periods in the frequency range $214 \mathrm{nHz}$ ( 54 days) to $38.5 \mathrm{nHz}$ (300 days). These periods are listed in Table 1 (long periods) and Table 2 (short periods). Many of these periodicities have previously been reported, but we discovered a few new periodicities in our analysis. These are listed in boldface in Tables 1 and 2 and are boxed in blue in Figures 4 and 7.

\subsection{The Low-Frequency Periods}

In the low-frequency range, $38.5 \mathrm{nHz}$ (300 days) to $2.6 \mathrm{nHz}$ (12 years), we restricted our analysis to periodicities in the range of 305 days to 5.6 years. Periods longer than 5.6 years 

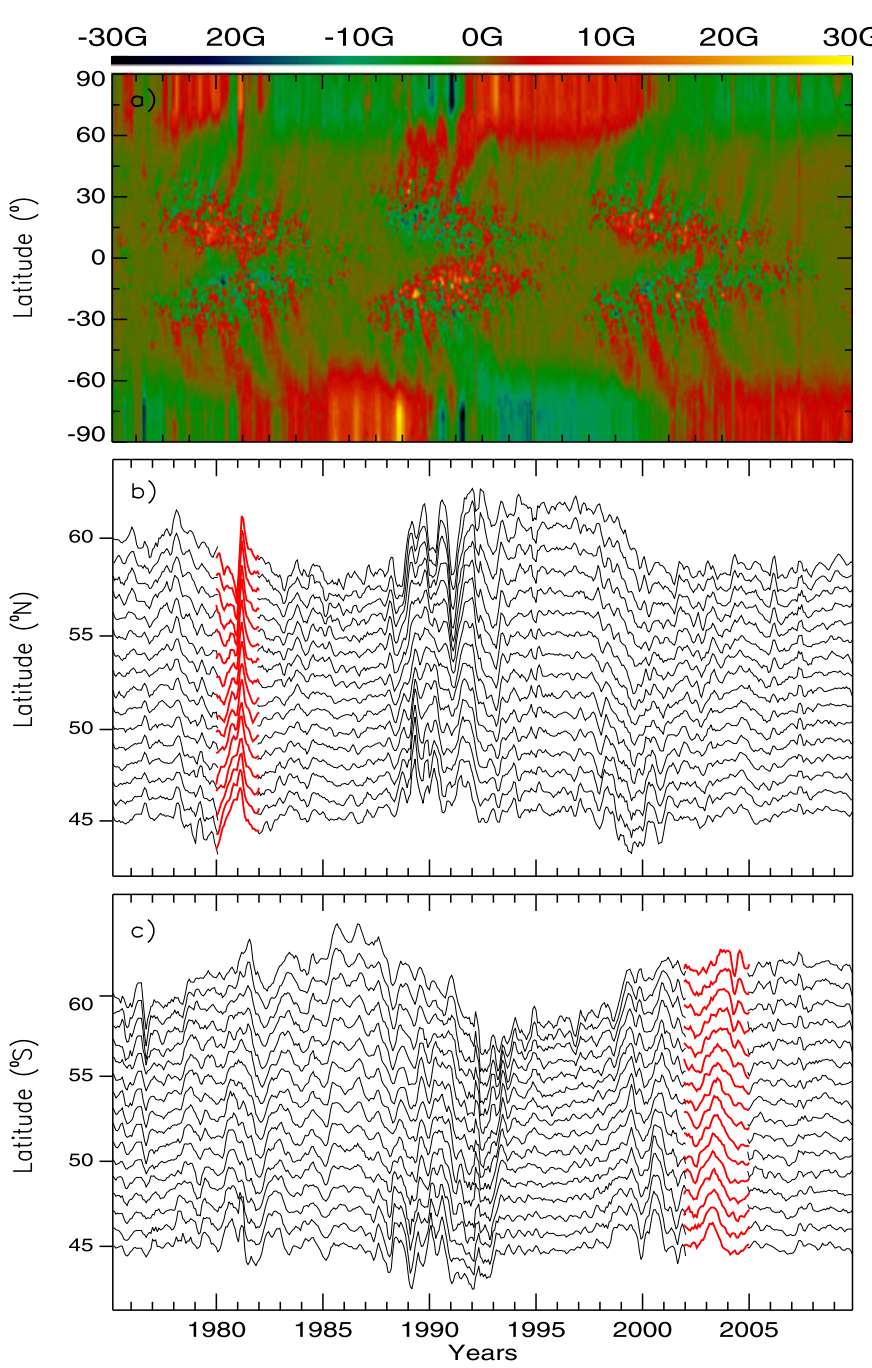

Figure 6 The top panel labeled (a) shows a magnetic butterfly-diagram generated using NSO/Kitt Peak magnetograms and depicts the net photospheric magnetic-flux distribution on the Sun for Solar Cycles 21, 22 , and 23. For better contrast, the magnetic flux has been limited to \pm 30 Gauss. The positive and negative polarities are shown in red and green. Polar surges or lateral motions of magnetic flux moving poleward above latitudes of $\pm 45^{\circ}$ are seen as tongues of red and green bands. The lower two panels labeled (b) and (c) show the variation of the magnetic field after smoothing over three Carrington rotations for each degree of latitude in the range of $45^{\circ}$ to $60^{\circ}$. An example of a surge is highlighted in red in the bottom two panels, representing the northern and southern hemisphere, respectively.

were excluded; they are inaccurately determined because we had to divide the time series. The periodicities are listed in Table 1, where the upper half lists periodicities for highlatitude fields in the North and South grouped before and after 1996, while the lower half lists periodicities for low-latitude fields in a similar fashion.

For photospheric fields in the high-latitude range we found periodicities of 305 days (0.84 years), 325 days (0.89 years), 339 days (0.92years), 341 days (0.93 years), 1 year, 
Table 1 The Fourier periods in the frequency range $38.5 \mathrm{nHz}$ to $2.6 \mathrm{nHz}$ (low-frequency zone), are listed with their Fourier power in the upper and lower half of the table for high- and low-latitude fields in the North and South before and after 1996.

\begin{tabular}{|c|c|c|c|c|c|c|c|}
\hline \multicolumn{4}{|c|}{ North high-latitude field } & \multicolumn{4}{|c|}{ South high-latitude field } \\
\hline \multicolumn{2}{|c|}{ Before 1996} & \multicolumn{2}{|l|}{ After 1996} & \multicolumn{2}{|c|}{ Before 1996} & \multicolumn{2}{|l|}{ After 1996} \\
\hline Period [yr] & Power & Period [yr] & Power & Period [yr] & Power & Period [yr] & Power \\
\hline 2.9 & 0.04 & 4.0 & 0.19 & 5.6 & 0.13 & 2.6 & 0.15 \\
\hline 2.3 & 0.04 & 2.0 & 0.08 & 4.0 & 0.05 & 1.5 & 0.04 \\
\hline 2.0 & 0.02 & 1.2 & 0.04 & 3.1 & 0.04 & 1.0 & 0.28 \\
\hline 1.4 & 0.02 & 1.0 & 0.16 & 2.4 & 0.05 & - & - \\
\hline 1.0 & 0.25 & 0.89 & 0.04 & 2.0 & 0.02 & - & - \\
\hline 0.93 & 0.06 & - & - & 1.5 & 0.04 & - & - \\
\hline- & - & - & - & 1.0 & 0.02 & - & - \\
\hline- & - & - & - & 0.92 & 0.03 & - & - \\
\hline- & - & - & - & 0.89 & 0.02 & - & - \\
\hline- & - & - & - & 0.84 & 0.04 & - & - \\
\hline - & - & - & - & - & - & - & - \\
\hline \multicolumn{4}{|c|}{ North low-latitude field } & \multicolumn{4}{|c|}{ South low-latitude field } \\
\hline \multicolumn{2}{|l|}{ Before 1996} & \multicolumn{2}{|l|}{ After 1996} & \multicolumn{2}{|l|}{ Before 1996} & \multicolumn{2}{|l|}{ After 1996} \\
\hline Period [yr] & Power & Period [yr] & Power & Period [yr] & Power & Period [yr] & Power \\
\hline 3.5 & 0.09 & 1.5 & 0.05 & 4.0 & 0.03 & 1.9 & 0.25 \\
\hline 2.5 & 0.10 & 1.0 & 0.04 & 3.1 & 0.09 & 1.1 & 0.04 \\
\hline 1.5 & 0.11 & 0.86 & 0.07 & 2.6 & 0.1 & 0.90 & 0.05 \\
\hline 1.3 & 0.04 & - & - & 1.8 & 0.05 & - & - \\
\hline 0.88 & 0.04 & - & - & 1.5 & 0.05 & - & - \\
\hline- & - & - & - & 1.44 & 0.04 & - & - \\
\hline- & - & - & - & 1.27 & 0.04 & - & - \\
\hline- & - & - & - & 0.94 & 0.04 & - & - \\
\hline - & - & - & - & 0.87 & 0.06 & - & - \\
\hline
\end{tabular}

1.2 years, 1.4 years, 1.5 years, 2 years, 2.4 years, 2.6 years, and 4 years, while for photospheric fields in the low-latitude range we found periods of 312 days ( 0.86 years), 318 days (0.87 years), 321 days ( 0.88 years), 327 days ( 0.9 years), and 343 days ( 0.94 years), 1 year, 1.27 years, 1.3 years, 1.44 years, 1.5 years, 1.8 years, 2.6 years, 3.5 years, and 4 years. All of these quasi-periodicities in various types of solar activity have been reported previously (Howe et al., 2000; Krivova and Solanki, 2002; Knaack, Stenflo, and Berdyugina, 2005; Mendoza, Velasco, and Valdés-Galicia, 2006; Chowdhury, Khan, and Ray, 2009). Knaack, Stenflo, and Berdyugina, 2005) had discussed a North-South asymmetry in quasi-periodic variations in unsigned photospheric flux with periods of 1.3 years, 1.5 years, and 2.6 years.

In addition to these known periodicities, we found some new periodicities in the highlatitude fields with periods of 2.3 years, 2.9 years, 3.1 years, and 5.6 years, and in the lowlatitude fields with periods 1.1 years, 1.9 years, 2.5 years, and 3.1 years. These new periodicities are shown in boldface in Tables 1 and 2. Although the periodicity of one year has been reported previously, we found this periodicity to be the prominent periodicity observed in both the North and South high-latitude fields before and after 1996. The Fourier power in the 
Table 2 The Fourier periods with their Fourier spectral power in the frequency range $214 \mathrm{nHz}$ to $38.5 \mathrm{nHz}$ (high-frequency zone) are listed in the upper and lower half of the table for high- and low-latitude fields in the North and South before and after 1996.

\begin{tabular}{|c|c|c|c|c|c|c|c|}
\hline \multicolumn{4}{|c|}{ North high-latitude field } & \multicolumn{4}{|c|}{ South high-latitude field } \\
\hline \multicolumn{2}{|l|}{ Before 1996} & \multicolumn{2}{|l|}{ After 1996} & \multicolumn{2}{|l|}{ Before 1996} & \multicolumn{2}{|l|}{ After 1996} \\
\hline Period [day] & Power & Period [day] & Power & Period [day] & Power & Period [day] & Power \\
\hline 294 & 0.02 & 253 & 0.04 & 288 & 0.037 & 184 & 0.07 \\
\hline 278 & 0.06 & 183 & 0.061 & 184 & 0.06 & - & - \\
\hline 263 & 0.04 & - & - & - & - & - & - \\
\hline 240 & 0.02 & - & - & - & - & - & - \\
\hline 183 & 0.04 & - & - & - & - & - & - \\
\hline 157 & 0.04 & - & - & - & - & - & - \\
\hline
\end{tabular}

\begin{tabular}{|c|c|c|c|c|c|c|c|}
\hline \multicolumn{4}{|c|}{ North low-latitude field } & \multicolumn{4}{|c|}{ South low-latitude field } \\
\hline \multicolumn{2}{|l|}{ Before 1996} & \multicolumn{2}{|l|}{ After 1996} & \multicolumn{2}{|l|}{ Before 1996} & \multicolumn{2}{|l|}{ After 1996} \\
\hline Period [day] & Power & Period [day] & Power & Period [day] & Power & Period [day] & Power \\
\hline 288 & 0.02 & 257 & 0.04 & 283 & 0.04 & - & - \\
\hline 278 & 0.03 & 181 & 0.08 & 248 & 0.02 & - & - \\
\hline 261 & 0.03 & 172 & 0.11 & 228 & 0.03 & - & - \\
\hline- & - & 147 & 0.04 & 157 & 0.03 & - & - \\
\hline- & - & - & - & 151 & 0.03 & - & - \\
\hline- & - & - & - & 145 & 0.03 & - & - \\
\hline- & - & - & - & - & - & - & - \\
\hline- & - & - & - & - & - & - & - \\
\hline
\end{tabular}

one-year period is comparatively higher in the North and South before 1996 than after 1996. On the other hand, although the one-year period is present in the low-latitude fields both in the North and South, it is only present after 1996. Additionally, periods of 1.2-1.6 years are common to both the North and South low-latitude fields and have significant power.

The periodicity most often discussed and reported is the 1.3-year periodicity (Krivova and Solanki, 2002; Knaack, Stenflo, and Berdyugina, 2004; Knaack, Stenflo, and Berdyugina, 2005). It has been linked to polar surges that can be seen most clearly in the latitude band of $45^{\circ}$ to $60^{\circ}$ (Wang, Nash, and Sheeley, 1989; Knaack, Stenflo, and Berdyugina, 2005) in the magnetic butterfly-diagrams, as depicted in Figure 6. The 1.3-year periodicity has also been linked to the variation of the rotation rate near the base of the convection zone (Howe et al., 2000).

\subsection{The High-Frequency Periods}

In the high-frequency range we have frequencies ranging from $214 \mathrm{nHz}$ (54 days) to $38.5 \mathrm{nHz}$ (300 days). These periodicities are listed in Table 2 for the high- and low-latitude fields. The upper half of Table 2 lists periodicities for the high-latitude fields with periods ranging between 157 days to 294 days. Some of these periodicities with their Fourier spectral power are shown in the top four panels (a), (b), (c), and (d) in Figure 7. Similarly, the lower half of Table 2 lists significant periodicities for the low-latitude fields with periods ranging between 134 days to 288 days. The bottom four panels (e), (f), (g), and (h) in 


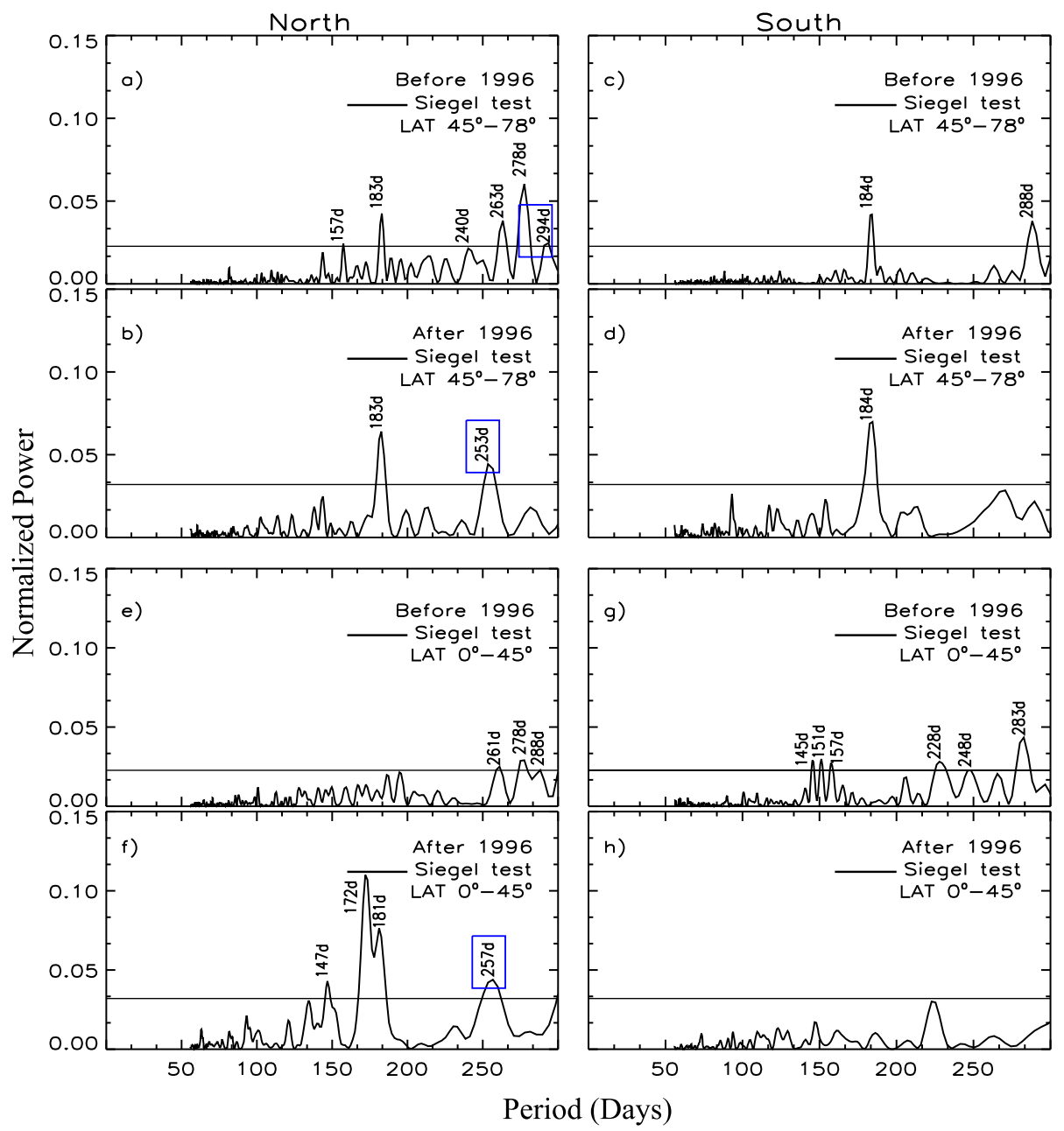

Figure 7 Normalized Fourier periodograms in the North (left column) and South (right column) before [(a), (c), (e), (g)] and after [(b), (d), (f), (h)] 1996 for high- (top panel) and low-latitude fields (bottom panel) for the high-frequency periods in the frequency range $214 \mathrm{nHz}$ to $38.5 \mathrm{nHz}$. The new periodicities from our analysis are boxed in blue.

Figure 7 show a few of these periodicities with their spectral power. As stated earlier, the significant periodic components were determined using the Siegel-test statistics; they are the periodic components with power levels above the black horizontal line drawn in each panel of Figure 7.

The known periodicities in the high-latitude zone include those with periods of 157 days, 183 days, 184 days, 240 days, 263 days, 278 days, and 288 days, and the new periodicities, viz. with periods of 253 days and 294 days not previously reported in the literature, are shown in boldface in the upper part of Table 2.

The Rieger periodicity of 157 days is a well-known fundamental periodicity that we found only in the high-latitude fields in the northern hemisphere prior to 1996. In addition, the periodicity of $182-184$ days is seen to be always present, and the Fourier power 
corresponding to this periodicity does not vary much in the North and South fields at high latitudes both before and after 1996. These high-frequency periods in the high-latitude fields do not show much variation in their normalized Fourier power, and we see no North-South asymmetry in these high-latitude fields.

On the other hand, in the low-latitude zone, shown in the lower part of Table 2, we report periodicities of 145 days, 147 days, 151 days, 157 days, 172 days, 228 days, 248 days, 261 days, 278 days, 283 days, and 288 days that have previously been reported (Oliver, Ballester, and Baudin, 1998; Krivova and Solanki, 2002; Knaack, Stenflo, and Berdyugina, 2005; Chowdhury, Khan, and Ray, 2009). The new periodicity from our analysis was 257 days and is shown in boldface in the lower half of Table 2. The Rieger periodicity of 157 days is only seen in the South low-latitude field before 1996. Likewise, the semi-annual period variation of 181 days is observed only in North low-latitude fields.

\section{Discussion and Conclusion}

Our wavelet analysis shows a transition occurring around 1996 in the distribution of power and periodicities of photospheric fields. When the data were divided into intervals prior to and after 1996, a hemispheric asymmetry became clear in the derived Fourier periodicities of the solar magnetic activity above latitudes of $\pm 45^{\circ}$. A more detailed analysis showed that this asymmetry is confined to the latitude band $45^{\circ}-60^{\circ}$ in both hemispheres. This latitude band is primarily dominated by strong, episodic poleward surges or tongues of magnetic flux in the two hemispheres, which, as stated earlier, are a direct surface manifestation of the meridional flow and the internal solar dynamo. In the $60^{\circ}-75^{\circ}$ latitude band we found an asymmetry in the distribution of spectral power in the longer periods because the photospheric surface fields have saturated in this band. The observed and localized asymmetry, which is confined to the $45^{\circ}-60^{\circ}$ latitude zone in the hemispheres, together with the fact that the solar fields and the micro-turbulence levels in the inner heliosphere have been decreasing since the early to mid-1990s suggests that active changes occurred around this time in the solar dynamo that governs the underlying basic processes in the Sun. These changes in turn probably initiated the build-up to one of the deepest solar minima, at the end of Cycle 23, experienced in the past 100 years.

The magnetic time-series in the two solar hemispheres exhibit a multitude of periodicities with significant variation in the spectral power of midterm (one-two years) periodicities before and after 1996. These prominent periods in the lower solar latitudes, below $\pm 45^{\circ}$, are thought to be generated by stochastic processes caused by the periodic emergence of surface magnetic flux (Wang and Sheeley, 2003) as the solar cycle progresses. As stated earlier, Howe et al. (2000) have reported a 1.3-year periodicity at the base of the solar convection zone, which has also been detected in sunspot areas and sunspot number time-series studied using wavelet transforms (Krivova and Solanki, 2002; Chowdhury and Dwivedi, 2011). These findings have led to the conclusion that the mid-term fluctuations in the solar fields are surface manifestations of changes in the magnetic fluxes generated deep inside the Sun.

Polar surges above latitudes $\pm 45^{\circ}$ show significant variations in their occurrence rates and strength in each cycle and are therefore manifested as a variation in spectral power and periods. A comparison of polar surges in the last three Solar Cycles, viz. 21, 22, and 23, revealed that they are somewhat stronger after the solar maximum.

Although we have restricted our analysis to data from Cycles 21, 22, and 23 in the period from $\approx 1975-2010$, an examination of data more recent than 2010 shows an asymmetry in 
Figure 8 The solar magnetic field in the northern (top) and southern (bottom) hemispheres in the latitude range of $45^{\circ}$ to $78^{\circ}$ for Solar Cycles 22 and 23. It is clear that the North solar field has reversed while the South solar field still has to do so. The reversal time of the North solar field is indicated by a small blue arrow.

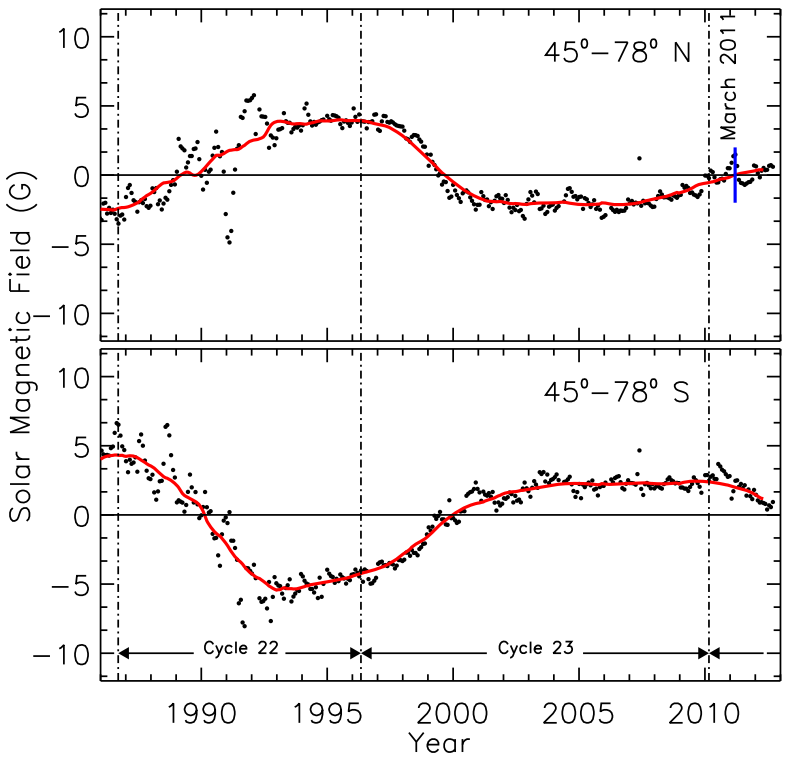

the solar field reversal at high latitudes. Figure 8 shows the reversal of the northern highlatitude field in Cycle 24 in March 2011. However, the southern hemisphere is yet to undergo a reversal. The filled black dots in Figure 8 are the actual measurements while the solid-red line is a smoothed curve. The reversal of the solar polar field occurs at times when the red curve passes through zero and is indicated for Cycle 24 by a thin blue line in the northern hemisphere. While it is known from earlier cycles that the two hemispheres do not reverse polarities at the same time, the time lag between the reversal of the northern and southern hemisphere has, to the best of our knowledge, never been as long.

In addition, Dikpati et al. (2010) and Dikpati (2011), using the theory of meridional circulation, have reported an asymmetry in the latitudinal extent of the Sun's meridional flow belt in Cycles 22 and 23, wherein surface meridional flows in Cycle 22 extend to latitudes of $\pm 60^{\circ}$, while in Cycle 23 they extended all the way to the Poles. Thus, the meridional flow in Cycle 23 took a longer time, thereby causing a slower return flow, which in turn led to the extended solar minimum in Cycle 23. Recent work, using helioseismic data from the Birmingham Solar Oscillation Network (BiSON), an instrument that is very sensitive in probing the solar interior close to the solar surface, has shown that the behavior of solar oscillation frequencies in the sub-surface magnetic layers during the descending phase of Cycle 23 was significantly different from that during Cycle 22 (Basu et al., 2012). Basu and co-authors went on to state that the peculiar solar minimum at the end of Cycle 23 could have been predicted long before it happened. The present work, showing a North-South asymmetry around the mid-1990s in the quasi-periodic variations of photospheric fields at high-latitudes, shows that changes were initiated at this time in the basic underlying solar dynamo processes such as the meridional flow rates and the magnetic-flux emergence that eventually led to the prolonged and deep minimum that we have just witnessed.

The current understanding of the solar dynamo is that it operates through the BabcockLeighton mechanism to produce poloidal fields through the decay of tilted bipolar sunspots (Choudhuri, Chatterjee, and Jiang, 2007; Jiang, Chatterjee, and Choudhuri, 2007). The strength of the polar field at each solar maximum then depends upon the tilt angle in bipolar sunspots which, in turn, is determined by the Coriolis force acting on magnetic-flux tubes 
that reach the surface by rising through the turbulent convection zone (D'Silva and Choudhuri, 1993). This process generates a large scatter in the average tilt-angle, causing the polar field to be randomly weaker or stronger than in the previous cycle (Longcope and Choudhuri, 2002). We have observed a decline in fields above $45^{\circ}$ for the past $\approx 15$ years, which implies that weak polar fields are generated in two successive solar cycles, viz. Cycles 22 and 23. A continuation of this declining trend beyond 22 years would imply a third successive weak polar field produced by the Babcock-Leighton mechanism. This is unlikely because the polar field is generated by a random process, and if this does happen, it would have serious implications for our current understanding of the solar dynamo. Moreover, two of the eight strongest geomagnetic storms in the last 150 years have occurred during Solar Cycles 13 and 14, which were both relatively weak cycles. Therefore, continued observations and measurements of solar magnetic fields are extremely important.

Acknowledgements NSO/Kitt Peak data used here produced cooperatively by NSF/NSO, NASA/GSFC, and NOAA/SEL. This work uses SOLIS data obtained by the NSO Integrated Synoptic Program (NISP), managed by the National Solar Observatory, which is operated by the Association of Universities for Research in Astronomy (AURA), Inc. under a cooperative agreement with the National Science Foundation. The wavelet software was provided by C. Torrence and G. Compo and is available at URL: atoc.colorado. edu/research/wavelets/

\section{References}

Ananthakrishnan, S., Balasubramanian, V., Janardhan, P.: 1995, Latitudinal variation of solar wind velocity. Space Sci. Rev. 72, 229-232. doi:10.1007/BF00768784.

Ananthakrishnan, S., Coles, W.A., Kaufman, J.J.: 1980, Microturbulence in solar wind streams. J. Geophys. Res. 85, 6025-6030. doi:10.1029/JA085iA11p06025.

Bai, T.: 1987, Periodicities of the flare occurrence rate in solar cycle 19. Astrophys. J. Lett. 318, L85-L91. doi:10.1086/184943.

Bai, T.: 2003, Periodicities in solar flare occurrence: analysis of cycles 19-23. Astrophys. J. 591, 406-415. doi:10.1086/375295.

Basu, S., Broomhall, A.-M., Chaplin, W.J., Elsworth, Y.: 2012, Thinning of the Sun's magnetic layer: the peculiar solar minimum could have been predicted. Astrophys. J. 758, 43. doi:10.1088/0004-637X/ $758 / 1 / 43$.

Choudhuri, A.R., Chatterjee, P., Jiang, J.: 2007, Predicting solar cycle 24 with a solar dynamo model. Phys. Rev. Lett. 98(13), 131103. doi:10.1103/PhysRevLett.98.131103.

Chowdhury, P., Dwivedi, B.N.: 2011, Periodicities of sunspot number and coronal index time series during solar cycle 23. Solar Phys. 270, 365 -383. doi:10.1007/s11207-011-9738-1.

Chowdhury, P., Khan, M., Ray, P.C.: 2009, Intermediate-term periodicities in sunspot areas during solar cycles 22 and 23. Mon. Not. Roy. Astron. Soc. 392, 1159-1180. doi:10.1111/j.1365-2966.2008.14117.x.

Chowdhury, P., Khan, M., Ray, P.C.: 2010, Short-term periodicities in sunspot activities during the descending phase of solar cycle 23. Solar Phys. 261, 173 - 191. doi:10.1007/s11207-009-9478-7.

Chowdhury, P., Ray, P.C.: 2006, Periodicities of solar electron flare occurrence: analysis of cycles 21-23. Mon. Not. Roy. Astron. Soc. 373, 1577 - 1589. doi:10.1111/j.1365-2966.2006.11120.x.

Dikpati, M.: 2011, Comparison of the past two solar minima from the perspective of the interior dynamics and dynamo of the Sun. Space Sci. Rev. 143. doi:10.1007/s11214-011-9790-z.

Dikpati, M., Gilman, P.A., de Toma, G., Ulrich, R.K.: 2010, Impact of changes in the Sun's conveyor-belt on recent solar cycles. Geophys. Res. Lett. 37, 14107. doi:10.1029/2010GL044143.

Droege, W., Gibbs, K., Grunsfeld, J.M., Meyer, P., Newport, B.J., Evenson, P., Moses, D.: 1990, A 153 day periodicity in the occurrence of solar flares producing energetic interplanetary electrons. Astrophys. $J$. Suppl. 73, 279-283. doi:10.1086/191463.

D'Silva, S., Choudhuri, A.R.: 1993, A theoretical model for tilts of bipolar magnetic regions. Astron. Astrophys. 272, 621 .

Fisher, R.A.: 1929, Tests of significance in harmonic analysis. Proc. Roy. Soc. London Ser. A, Math. Phys. Sci. 125, 54-59.

Forrest, D.J., Chupp, E.L., Ryan, J.M., Cherry, M.L., Gleske, I.U., Reppin, C., Pinkau, K., Rieger, E., Kanbach, G., Kinzer, R.L.: 1980, The gamma ray spectrometer for the Solar Maximum Mission. Solar Phys. 65, 15-23. doi:10.1007/BF00151381. 
Hathaway, D.H., Rightmire, L.: 2010, Variations in the Sun's meridional flow over a solar cycle. Science 327, 1350. doi:10.1126/science.1181990.

Howard, R.: 1974, Studies of solar magnetic fields. II - The magnetic fluxes. Solar Phys. 38, $59-67$. doi:10.1007/BF00161823.

Howe, R., Christensen-Dalsgaard, J., Hill, F., Komm, R.W., Larsen, R.M., Schou, J., Thompson, M.J., Toomre, J.: 2000, Dynamic variations at the base of the solar convection zone. Science 287, 24562460. doi:10.1126/science.287.5462.2456.

Janardhan, P., Bisoi, S.K., Gosain, S.: 2010, Solar polar fields during cycles 21 -23: correlation with meridional flows. Solar Phys. 267, 267 -277. doi:10.1007/s11207-010-9653-x.

Janardhan, P., Bisoi, S.K., Ananthakrishnan, S., Tokumaru, M., Fujiki, K.: 2011, The prelude to the deep minimum between solar cycles 23 and 24: interplanetary scintillation signatures in the inner heliosphere. Geophys. Res. Lett. 382, 20108. doi:10.1029/2011GL049227.

Jian, L.K., Russell, C.T., Luhmann, J.G.: 2011, Comparing solar minimum 23/24 with historical solar wind records at 1 AU. Solar Phys. 69. doi:10.1007/s11207-011-9737-2.

Jiang, J., Chatterjee, P., Choudhuri, A.R.: 2007, Solar activity forecast with a dynamo model. Mon. Not. Roy. Astron. Soc. 381, 1527 - 1542. doi:10.1111/j.1365-2966.2007.12267.x.

Jiang, J., Cameron, R.H., Schmitt, D., Schussler, M.: 2011, Can surface flux transport account for the weak polar field in cycle 23? Space Sci. Rev. 136. doi:10.1007/s11214-011-9783-y.

Kile, J.N., Cliver, E.W.: 1991, A search for the 154 day periodicity in the occurrence rate of solar flares using Ottawa 2.8 GHz burst data, 1955 - 1990. Astrophys. J. 370, 442 - 448. doi:10.1086/169831.

Kiliç, H.: 2008, Midrange periodicities in sunspot numbers and flare index during solar cycle 23. Astron. Astrophys. 481, 235 - 238. doi:10.1051/0004-6361:20078455.

Kirk, M.S., Pesnell, W.D., Young, C.A., Hess Webber, S.A.: 2009, Automated detection of EUV polar coronal holes during solar cycle 23. Solar Phys. 257, 99 - 112. doi:10.1007/s11207-009-9369-y.

Knaack, R., Stenflo, J.O., Berdyugina, S.V.: 2004, Periodic oscillations in the North-South asymmetry of the solar magnetic field. Astron. Astrophys. 418, L17 - L20. doi:10.1051/0004-6361:20040107.

Knaack, R., Stenflo, J.O., Berdyugina, S.V.: 2005, Evolution and rotation of large-scale photospheric magnetic fields of the Sun during cycles $21-23$. periodicities, North-South asymmetries and r-mode signatures. Astron. Astrophys. 438, 1067 - 1082. doi:10.1051/0004-6361:20042091.

Krivova, N.A., Solanki, S.K.: 2002, The 1.3-year and 156-day periodicities in sunspot data: wavelet analysis suggests a common origin. Astron. Astrophys. 394, 701 - 706. doi:10.1051/0004-6361:20021063.

Lean, J.: 1990, Evolution of the 155 day periodicity in sunspot areas during solar cycles 12 to 21. Astrophys. J. 363, $718-727$. doi: $10.1086 / 169378$.

Livingston, W.: 2002, Sunspots observed to physically weaken in 2000-2001. Solar Phys. 207, 41 - 45.

Lomb, N.R.: 1976, Least-squares frequency analysis of unequally spaced data. Astrophys. Space Sci. 39, 447 - 462. doi:10.1007/BF00648343.

Longcope, D., Choudhuri, A.R.: 2002, The orientational relaxation of bipolar active regions. Solar Phys. 205, 63 - 92. doi:10.1023/A:1013896013842.

McComas, D.J., Ebert, R.W., Elliott, H.A., Goldstein, B.E., Gosling, J.T., Schwadron, N.A., Skoug, R.M.: 2008, Weaker solar wind from the polar coronal holes and the whole Sun. Geophys. Res. Lett. 35, L18103. doi:10.1029/2008GL034896.

Mendoza, B., Velasco, V.M., Valdés-Galicia, J.F.: 2006, Mid-term periodicities in the solar magnetic flux. Solar Phys. 233, 319-330. doi:10.1007/s11207-006-4122-2.

Nandy, D., Muñoz-Jaramillo, A., Martens, P.C.H.: 2011, The unusual minimum of sunspot cycle 23 caused by meridional plasma flow variations. Nature 471, 80-82. doi:10.1038/nature09786.

Oliver, R., Ballester, J.L.: 1994, The North-South asymmetry of sunspot areas during solar cycle 22. Solar Phys. 152, 481 - 485. doi:10.1007/BF00680451.

Oliver, R., Ballester, J.L., Baudin, F.: 1998, Emergence of magnetic flux on the Sun as the cause of a 158-day periodicity in sunspot areas. Nature 394, $552-553$. doi:10.1038/29012.

Penn, M.J., Livingston, W.: 2006, Temporal changes in sunspot umbral magnetic fields and temperatures. Astrophys. J. Lett. 649, L45 - L48. doi:10.1086/508345.

Percival, D.B., Walden, A.T.: 1993, Spectral Analysis for Physical Applications, Cambridge University Press, Cambridge.

Rieger, E., Kanbach, G., Reppin, C., Share, G.H., Forrest, D.J., Chupp, E.L.: 1984, A 154-day periodicity in the occurrence of hard solar flares? Nature 312, 623-625. doi:10.1038/312623a0.

Savitzky, A., Golay, M.J.E.: 1964, Smoothing and differentiation of data by simplified least squares procedures. Anal. Chem. 36, $1627-1639$.

Scargle, J.D.: 1982, Studies in astronomical time series analysis. II - Statistical aspects of spectral analysis of unevenly spaced data. Astrophys. J. 263, 835-853. doi:10.1086/160554.

Scargle, J.D.: 1989, Studies in astronomical time series analysis. III - Fourier transforms, autocorrelation functions, and cross-correlation functions of unevenly spaced data. Astrophys. J. 343, 874-887. doi:10.1086/167757. 
Schultz, M., Stattegger, K.: 1997, Spectrum: spectral analysis of unevenly spaced paleoclimatic time series. Comp. Geosci. 23, $929-945$.

Siegel, A.F.: 1980, Testing for periodicity in a time series. J. Am. Stat. Assoc. 75, $345-348$.

Torrence, C., Compo, G.P.: 1998, A practical guide to wavelet analysis. Bull. Am. Meteorol. Soc. 79, $61-78$.

Verma, V.K.: 1987, On the increase of solar activity in the Southern Hemisphere during solar cycle 21. Solar Phys. 114, $185-188$.

Verma, V.K., Joshi, G.C.: 1987, On the periodicities of sunspots and solar strong hard X-ray bursts. Solar Phys. 114, 415 - 418. doi:10.1007/BF00167358.

Wang, Y.-M., Sheeley, N.R. Jr.: 2003, On the fluctuating component of the Sun's large-scale magnetic field. Astrophys. J. 590, $1111-1120$. doi:10.1086/375026.

Wang, Y.-M., Nash, A.G., Sheeley, N.R. Jr.: 1989, Evolution of the Sun's polar fields during sunspot cycle 21 - poleward surges and long-term behavior. Astrophys. J. 347, 529-539. doi:10.1086/168143.

Wang, Y.-M., Robbrecht, E., Sheeley, N.R. Jr.: 2009, On the weakening of the polar magnetic fields during solar cycle 23. Astrophys. J. 707, 1372 - 1386. doi:10.1088/0004-637X/707/2/1372.

Welch, P.D.: 1967, The use of fast Fourier transform for the estimation of power spectra: a method based on time averaging over short, modified periodograms. IEEE Trans. Audio Electroacoust. 15, 70-73. 